\title{
Cherenkov radiation from electrons passing through human tissue
}

\author{
Pavel Lukin**, Izmail Batkin ${ }^{\dagger}$, Alexandr N. Almaliev*, Tatyana A. Churakova*, \\ Mikhail A. Dolgopolov* and Igor V. Kopytin* \\ *Department of Physics, Voronezh State University \\ 1 Universitetskaya Sq., Voronezh 394000, Russia \\ ${ }^{\dagger}$ School of Electrical Engineering and Computer Science \\ University of Ottawa \\ 800 King Edward Avenue, Ottawa, ON Canada, K1N 6N5 \\ †ukin_pv@phys.vsu.ru
}

Received 4 August 2016

Accepted 28 September 2016

Published 14 November 2016

\begin{abstract}
A method for investigating the optical properties of human tissues is suggested. The method is based on the measurement of Cherenkov radiation produced by relativistic electrons passing through the tissue. Monte-Carlo simulation of visible photon emission and propagation is carried out taking into account multiple electron and photon scattering processes. Sensitivity of the Cherenkov radiation to the optical characteristics of human tissues is demonstrated.
\end{abstract}

Keywords: Coherent radiation; human tissue optics; multiple scattering.

\section{Introduction}

The tissues of human body are translucent to visible light in the red and infrared region. This optical property has been used to develop a new method for diagnosing and treating some diseases. ${ }^{1}$ The actual experimental investigation of the optical features of human tissue have been carried out in several works. For example, measurement of the angular distribution of the transmitted and reflected light from an optical source located outside the human tissue have been done. ${ }^{2,3}$
The main difficulties of these studies are associated with the large elastic scattering ability of human tissue. Typically, the free path of light photons with wavelength $600-1000 \mathrm{~nm}$ in human tissue ranges from 0.01 to $0.1 \mathrm{~mm} .{ }^{4} \mathrm{In}$ order to determine refraction index, absorption coefficients, albedo and other optical features, it is necessary to use extremely thin layers of tissue, and locate photodetector at different angles, considering the incident angles of the initial light beam. However, preparing tissue samples reduces the accuracy of the

\$Corresponding author.

This is an Open Access article published by World Scientific Publishing Company. It is distributed under the terms of the Creative Commons Attribution 4.0 (CC-BY) License. Further distribution of this work is permitted, provided the original work is properly cited. 
experimental data, and complex optical systems do not allow "in vivo" measurements of the optical characteristics.

The application of the Cherenkov light for radiation therapy dosimetry has been discussed and analyzed in several recent publications. ${ }^{5-10}$ It was concluded that the Cherenkov emission is suitable for surface dosimetry applications. ${ }^{7}$

In the present paper, we suggest the use of Cherenkov radiation produced by relativistic electrons for investigation of optical properties of human tissues. Since the features of Cherenkov radiation are well known, use of the Cherenkov radiation as a source of visible photons for the investigation of human tissue has several advantages:

(1) The intensity and spectrum of the emitted photons are a simple function of the refraction index.

(2) Cherenkov radiation is an internal source of photons. Hence, there is no difficulty associated with photon's reflection at the tissue surface.

(3) It is possible to vary the "position" of the produced Cherenkov source of light by changing the electron's energy.

(4) It is possible to generate short light pulses in order to distinguish the true signal against the background.

(5) It is possible to investigate the spatial distribution of optical characteristics in the human tissue.

(6) Alternatively, if the optical properties of the human tissue are known, it is possible to study electron's passage through the media.

In this paper, we carry out the theoretical investigation of Cherenkov light emission by an electron passing through human tissue. A numerical statistical simulation method is used to study the propagation of electrons and photons in human tissues, taking into account multiple electron and photon scattering processes. The theoretical model is described in Sec. 2. The results of the numerical simulation are discussed in Sec. 3.

\section{Theoretical Model}

\subsection{Generating light photons by the electrons}

Let us assume that the refraction index of human tissue is $n(\omega)$, where $\omega$ is the frequency of the light.
Typical values of $n(\omega)$ for photons with wavelengths $600-1200 \mathrm{~nm}$ are $1.3-1.5 .^{4}$ The energy of Cherenkov radiation emitted by an electron with energy $E_{e}$ traveling a distance $d x$ is given by the formula ${ }^{11}$ :

$$
\frac{d W}{d x}=\left(\frac{e}{c}\right)^{2} \int\left[1-\frac{1}{(\beta n(\omega))^{2}}\right] \omega d \omega,
$$

where the direction of propagation of photons is defined by the angle $\theta_{\gamma}$ with respect to the direction of the electron momentum such that

$$
\cos \left(\theta_{\gamma}\right)=\frac{1}{\beta n(\omega)},
$$

where $\beta=v / c$ is the relativistic electron velocity.

Let us consider Cherenkov radiation produced by an electron beam with an initial energy of $E_{e}^{0}$. For simplicity, we assume that the target is a slab of thickness $D$. In order to carry out a statistical simulation of an electron traveling through matter, we use the Moliere theory of multiple scattering. ${ }^{12,13}$ According to Ref. 12 we can describe the angular distribution $F(\theta)$ of electrons with energy $E_{e}$ traveling a distance $T$ in the slab, by the formula:

$$
\begin{aligned}
F(\Theta) 2 \pi \Theta d \Theta= & {\left[2 \exp \left(-\psi^{2}\right)+\frac{F_{1}(\psi)}{B}\right.} \\
& \left.+\frac{F_{2}(\psi)}{B^{2}}+\cdots\right] \psi d \psi,
\end{aligned}
$$

where $\Theta$ is the angle between the initial and final directions of the electron, and $\psi$ is an internal dummy angle related to $\Theta$ as follows:

$$
\psi=\frac{\Theta}{\chi_{e} \sqrt{B}},
$$

where the functions $F_{1}, F_{2}, \chi_{e}$, and $B$ depend on the electron energy $E_{e}$, the slab thickness $T$, the charge number $Z$, the atomic number $A$, and the density of the target $\rho$. Numerical values of the functions $F_{1}$ and $F_{2}$ are tabulated in Ref. 12 .

First, we calculate the maximum possible range for an electron with energy $E_{e}^{0}$ in human tissue, using the equation:

$$
L_{e}=\int \frac{d E_{e}}{\left(-\frac{d E}{d x}\right)},
$$


where the continuous energy losses $(-d E / d x)$ is given by the formula ${ }^{12}$ :

$$
\begin{aligned}
\left(-\frac{d E}{d x}\right)= & 0.153 \rho \frac{Z}{A \beta^{2}}\left[\ln \left(\frac{E\left(E+m c^{2}\right)^{2} \beta^{2}}{2 I^{2}\left(m c^{2}\right)}\right)\right. \\
& \left.-\left(2 \sqrt{1-\beta^{2}}-1+\beta^{2}\right) \ln 2+\left(1-\beta^{2}\right)\right] .
\end{aligned}
$$

In order to satisfy the conditions claimed by Moliere's theory, ${ }^{12}$ we divide the maximum range $L_{e}$ into $N$ different layers each of thickness $T$

$$
T=\frac{L}{N_{l}},
$$

where $N_{l}$ is varied from 20 to 50 depending on the initial energy of the electron.

The algorithm of the statistical simulation consists of a sequence of steps for each layer. Considering the $i$ th layer, we start by calculating the energy $E_{i}$ and the momentum $P_{i}$ of the electron as it passes through the layer. Then, we determine the number of Cherenkov photons with energies ranging from $E_{\gamma}$ to $E_{\gamma}+d E_{\gamma}$ emitted by the electron in that layer:

$$
\frac{d N}{d E_{\gamma}}=\left(\frac{e}{c}\right)^{2} T \int\left[1-\frac{1}{(\beta n(\omega))^{2}}\right] \frac{\omega d \omega}{E_{\gamma}} .
$$

The Cherenkov photons are emitted at an angle $\theta_{\gamma}$ with respect to the momentum direction of the electron (see Eq. (2)), but with different azimuthal angles $\phi_{\gamma}$, chosen via

$$
\phi_{\gamma}=2 \pi \times \eta,
$$

where $\eta$ is a uniformly distributed random number on the interval $[0,1]$. Finally, we watch the fate of each Cherenkov photon created in that layer.

\subsection{The propagation of light in human tissue}

To study all optical properties of human tissue is a very complicated task. However, a simple model can be used to describe the propagation of unpolarized light in human tissues. ${ }^{4}$ In this model, optical properties can be described in terms of the refractive index $n(\omega)$, the total attenuation coefficient $\Sigma(\omega)$, albedo $a(\omega)$, and the mean cosine of the photon scattering angle $g(\omega)$.
In diffused media, the angular distribution of the scattered photons is given by the Henyey-Greenstein formula ${ }^{14}$ :

$$
S\left(\theta_{s}\right)=\frac{0.5\left(1-g^{2}\right)}{\sqrt{\left(1+g^{2}-2 g \cos \left(\theta_{s}\right)\right)^{3}}},
$$

where $\theta_{s}$ is the scattering angle. Recall that $g$ is defined as mean of $\cos \left(\theta_{s}\right)$. The total attenuation coefficient $\Sigma$ is defined as the sum of the attenuation coefficients for scattering $\left(\Sigma_{s}\right)$ and the absorption $\left(\Sigma_{a}\right)$ processes:

$$
\Sigma=\Sigma_{s}+\Sigma_{a},
$$

where $\Sigma_{s}=1 / L_{s}$, an $L_{s}$ is the photon mean free path for scattering, $\Sigma_{a}=1 / L_{a}, L_{a}$ is the photon mean free path for the absorption. Albedo is given by

$$
a=\frac{\Sigma_{s}}{\Sigma_{s}+\Sigma_{a}}=\frac{L_{a}}{L_{a}+L_{s}} .
$$

The parameters $n(\omega), \Sigma, a$, and $g$ depend on the photon energy and the type of tissue. We would like to note that inelastic photon scattering has not been taken into account. In fact, in the wavelength range under consideration $(600-1200 \mathrm{~nm})$, the probability of inelastic photon scattering is rather small.

To simulate the travel of Cherenkov photons through a diffused media we use the Monte-Carlo method. Firstly, we use the procedure described in Sec. 2.1 to calculate the initial conditions (the number, coordinates, and direction of propagation) of Cherenkov photons emitted by the electrons. Then, using the random number generator, we determine the coordinates of the scattering (or absorbing) center. Finally, the Henyey-Greenstein angular distribution (Eq. (10)) is used to calculate the scattering angle $\theta_{\gamma}$. This procedure is repeated until the photon will be absorbed or escaped from the slab.

\section{Results and Discussion}

Figures 1-6 show the results of the numerical simulations for the number of photons of energy between $1-2 \mathrm{eV}$, resulting from one electron, and their dependence on the initial electron energy $E_{e}^{0}$, the attenuation coefficient $\Sigma$, the mean cosine $g$, and the thickness of the slab $D$. We point out that the electron source is located outside of the slab and that the values of some of these optical parameters are chosen to be within the typical range of values of human tissue for red and infrared light. ${ }^{2}$ 


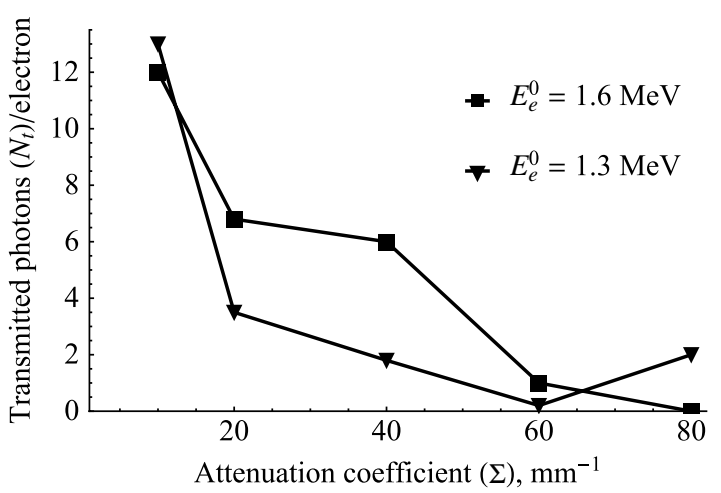

Fig. 1. Intensity of transmitted photons versus the attenuation parameter, for $E_{e}^{0}=1.6 \mathrm{MeV}$ and $E_{e}^{0}=1.3 \mathrm{MeV}(a=0.99$, $g=0.98, D=5.0 \mathrm{~mm}$ ).

For example, we have fixed the albedo $a=0.99$, and the refraction coefficient $n(\omega)=1.33$. For simplicity, we do not take into account the dependence of the optical characteristic on $\omega$.

Figures 1 and 2 demonstrate the dependence of the number of transmitted photons $N_{t}$ and reflected photons $N_{r}$ per electron on the attenuation coefficient $\Sigma$. It can be seen that the number of transmitted and reflected photons are rather sensitive to the attenuation coefficient $\Sigma$.

Figure 3 shows that the number of photons transmitted through the target is enhanced as the mean cosine of the scattering angle increases while the number of reflected photons decreases. It should be recalled that in the case under simulation, Cherenkov photons are emitted by the electron beam which is normal to the surface of the slab.

Figure 4 illustrates the strong dependence of the number of photons before and after the target on the target thickness.

Finally, Figs. 5 and 6 demonstrate the sensitivity of the number of transmitted and reflected photons

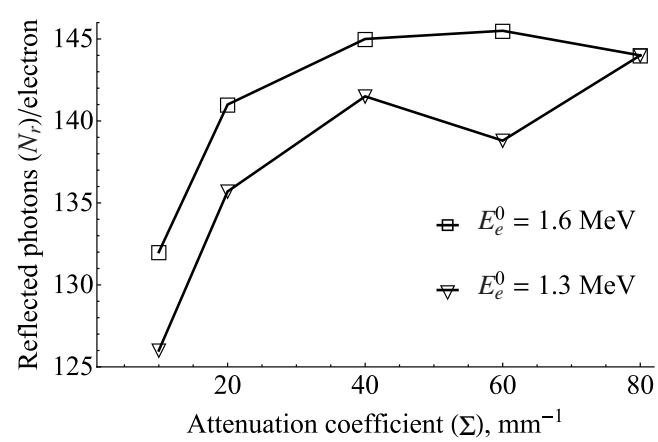

Fig. 2. Intensity of reflected photons versus the attenuation parameter, for $E_{e}^{0}=1.6 \mathrm{MeV}$ and $E_{e}^{0}=1.3 \mathrm{MeV} \quad(a=0.99$, $g=0.98, D=5.0 \mathrm{~mm}$ ).

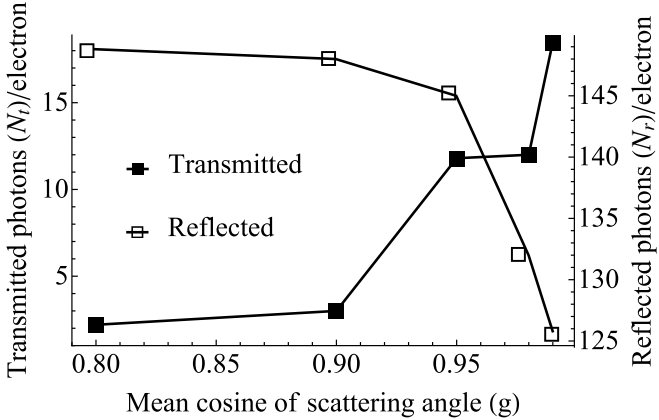

Fig. 3. Dependence of the number of transmitted and reflected photons on the mean cosine of scattering angle $\left(E_{e}^{0}=1.6 \mathrm{MeV}\right.$, $\Sigma=10.0 \mathrm{~mm}^{-1}, a=0.99, D=5.0 \mathrm{~mm}$ ).

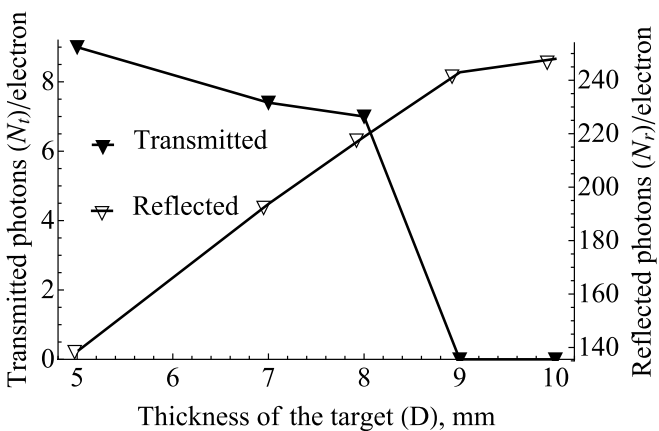

Fig. 4. Dependence of the number of transmitted and reflected photons on the thickness of the target $\left(E_{e}^{0}=1.9 \mathrm{MeV}\right.$, $\left.\Sigma=10.0 \mathrm{~mm}^{-1}, a=0.99, g=0.98\right)$.

on the energy of the incident electron for two slab thicknesses.

The results demonstrate that the number of transmitted and reflected photons per electron with energy $1-2 \mathrm{MeV}$ range from 1 to 200. Modern photomultiplier tubes exhibit quantum efficiencies of greater than $10 \%$. Let us assume that about $10^{5}$ electrons penetrate into the human tissue. In this

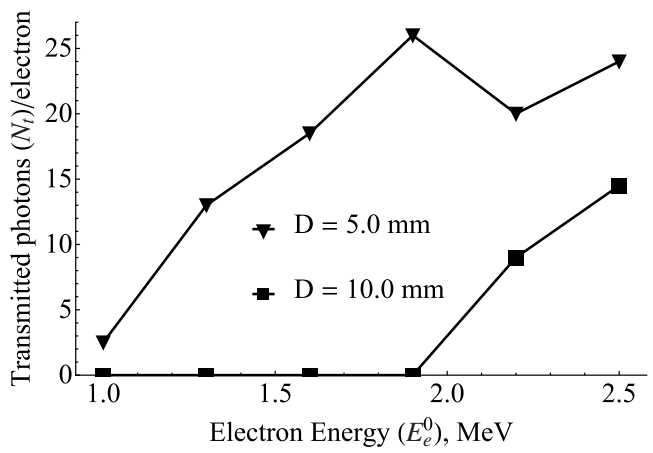

Fig. 5. Transmitted photons through a slab of thickness $D=$ $5.0 \mathrm{~mm}$ and $D=10.0 \mathrm{~mm}$ and their dependence on the electron incident energy $\left(\Sigma=10.0 \mathrm{~mm}^{-1}, a=0.99, g=0.98\right)$. 


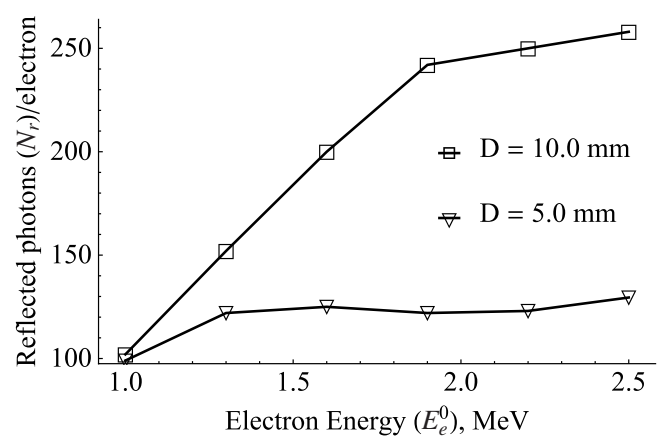

Fig. 6. Reflected photons from a slab of thickness $D=5.0 \mathrm{~mm}$ and $D=10.0 \mathrm{~mm}$ and their dependence on the electron incident energy $\left(\Sigma=10.0 \mathrm{~mm}^{-1}, a=0.99, g=0.98\right)$.

case, about $10^{6}$ photons will be emitted. This amount will be enough to be detected with high statistical accuracy. It is easy to estimate that $10^{5}$ electrons with average energy $1.5 \mathrm{MeV}$ will create equivalent dose less $20 \mu G y$. This equivalent dose is far less than that annual dose due to natural radioactivity. For this reason, the suggested method may be applied for "in vivo" measurements of the optical features of human tissue.

\section{Conclusions}

The possibility of a new method for studying the optical properties of human tissue using Cherenkov emission of visible or infrared light by relativistic electrons has been investigated.

Statistical simulation shows that the number of photons before and after the target is rather highly sensitive to the optical properties of human tissue. Using this fact, it is possible to measure the intensity of the Cherenkov photons produced by relativistic electrons with different energies to determine the optical constants of human tissue, and vice versa.

We suggest that this method could be utilized to serve as an important source of information about the optical properties of human tissue. We suggest that this method could be served as an important source of information about optical properties of human tissue as well as about radiation dose from relativistic electrons.

\section{References}

1. H. Wallberg, "Diaphanography in various breast disorders," Acta Radiol. Diagn. 26, 271-276 (1985).

2. V. G. Peters, D. R. Wymant, M. S. Patterson, G. L. Frank, "Optical properties of normal and diseased breast human tissues in the visible and near infrared," Phys. Med. Biol. 35, 1317-1334 (1990).

3. B. C. Wilson, S. L. Jacques, "Optical reflectance and transmittance of tissue: Principles and applications," IEEE J. Quantum Electron. 26, 2186-2199 (1990).

4. W. F. Cheong, S. A. Prahl, A. J. Welch, "A review of the optical properties of biological tissues," IEEE J. Quantum Electron. 26, 2166-2185 (1990).

5. S. L. Jacques, "Optical properties of biological tissues: a review," Phys. Med. Biol. 58, R37-R61 (2013).

6. J. Axelsson, A. K. Glaser, D. J. Gladstone, B. W. Pogue, "Quantitative Cherenkov emission spectroscopy for tissue oxygenation assessment," Opt. Express 20(5), 5133 (2012).

7. A. K Glaser, R. Zhang, D. J. Gladstone, B. W. Pogue, "Optical dosimetry of radiotherapy beams using Cherenkov radiation: The relationship between light emission and dose," Phys. Med. Biol. 59, 3789-3811 (2014).

8. X. Ma, J. Wang, Z. Cheng, "Cerenkov radiation: A multi-functional approach for biological sciences," Front. Phys. 2(4) (2014). http://dx.doi.org/ 10.3389/fphy.2014.00004.

9. A. E. Spinelli, M. Ferdeghini, C. Cavedon, E. Zivelonghi, R. Calandrino, A. Fenzi, A. Sbarbati, F. Boschi, "First human Cerenkography," J. Biomed. Opt. 18(2), 020502 (2013).

10. Y. Helo, I. Rosenberg, D. D'Souza, L. MacDonald, R. Speller, G. Royle, A. Gibsons, "Imaging Cerenkov emission as a quality assurance tool in electron radiotherapy," Phys. Med. Biol. 59, 1963-1978 (2014).

11. V. P. Zrelov, Cherenkov Radiation in High-Energy Physics, 1st Edition, B. Baruch, Ed., Part 1, Chap. 2, p. 66, The U.S. Atomic Energy Commission and The National Science Foundation, Washington, D.C. (1970).

12. G. Knop, W. Paul, Interaction of electrons and $\alpha-$ particles with matter, $\alpha-, \beta-, \gamma-$ Ray Spectroscopy Charp. 1, K. Siegbahn, Ed., North-Holland Publishing Company, Amsterdam (1965).

13. W. R. Nelson, H. Hirayama, D. W. O. Rogers, The EGS4 Code System, SLAC-Report-265, Stanford Linear Accelerator Center (1985).

14. L. G. Henyey, J. L. Greenstein, "Diffuse radiation in the galaxy," Astrophys. J. 93, 70-83 (1941). 